\title{
Modélisation tridimensionnelle des plasmas thermiques en écoulement
}

\author{
P. L. Viollet, M. Gabillard et N. Méchitoua \\ EDF, Laboratoire National d'Hydraulique, 6 Quai Watier, 78400 Chatou, France
}

(Reçu le 18 décembre 1989, révisé le 25 avril 1990, accepté le 16 mai 1990)

\begin{abstract}
Résumé. - Parmi les difficultés de modélisation des écoulements de plasma thermique en excitation, la turbulence et son interaction avec les phénomènes réactifs est l'une des plus aigues. Dans le cas où le plasma et le courant de dilution sont produits à partir du même gaz, cas qui minimise cette difficulté, une démarche de validation de la modélisation numérique a été effectuée. Cette démarche comprend des cas de validation en écoulement incompressible, en écoulement non-réactif à masse volumique variable, enfin des cas de mélange plasma-courant de dilution. Dans le cas des réacteurs chimiques à plasma, le problème de l'intéraction entre la turbulence et les réactions chimiques se pose de façon plus aigue, et nous avons tenté de discuter ici de quelques aspects de ce problème.
\end{abstract}

\begin{abstract}
One of the main difficulties in modelling thermal plasma flows is the interaction between turbulence and reacting phenomena. In the less difficult case where both plasma and diluting flow are produced with the same gas, a validation study of a numerical modelling has been done. This validation study consists in incompressible flow situations, non-reacting flows with large density differences, and thermal plasma flows mixing with cold flow. In the case of plasma chemical reactors, the paper discusses some aspects of the problem of interaction between turbulence and chemical reactions.
\end{abstract}

\section{Introduction.}

Un plasma en extinction se mélangeant avec un écoulement de dilution froid est un écoulement souvent rencontré dans les applications industrielles. Dans les applications de chauffage de gaz par plasma, qui ont été beaucoup explorées ces derniers temps, le gaz plasmagène et le gaz froid de dilution sont identiques, ce qui simplifie notablement le problème. Dans les applications à venir aux réacteurs chimiques à plasma, en revanche, on pourra rencontrer un plasma produit à partir d'un gaz $A_{1}$ réagissant avec un autre gaz $A_{2}$, par exemple un plasma d'hydrogène-argon réagissant avec une injection de méthane.

Du point de vue de la mécanique des fluides, les écoulements résultant sont essentiellement des écoulements turbulents subsoniques, présentant de grandes différences de masse volumique (le rapport des densités $\mathbb{R}$ entre le plasma, généralement émis sous la forme d'un jet turbulent et l'écoulement de dilution peut dépasser largement 10). De tels types d'écoulements sont également rencontrés dans un certain nombre de problèmes industriels, problèmes de combustion et de propulsion $(\mathbb{R} \sim 5)$, de mélanges de gaz, etc...
Le présent papier a d'abord pour objectif d'analyser les problèmes de modélisation posés par ces écoulements (paragraphe 2), notamment en ce qui concerne l'influence de la turbulence. On présente ensuite, au paragraphe 3 , la démarche de validation des logiciels tridimensionnels qui sont appliqués à ce type d'écoulements, en se restreignant aux problèmes dans lesquels la composition atomique est constante (le plasma obtenu à partir du gaz $\mathrm{N}$ se mélange avec ce même gaz $\mathrm{N}$ froid). L'analyse d'une flamme de diffusion turbulente permet par ailleurs une première validation concernant un écoulement réactif de composition atomique variable.

\section{Sur la modélisation d'un écoulement turbulent ré-} actif multiconstituants.

\subsection{EQUATIONS GÉNÉRALES D'UN ÉCOULEMENT RÉ-} ACTIFÀ PLUSIEURS CONSTITUANTS. - Nous ne considérons ici que les phénomènes ayant lieu en phase gazeuse, en écrivant les équations de bilans des espèces et de l'énergie de la phase gazeuse, pouvant contenir des inclusions dispersées liquides (goutelettes) ou 
solides (particules). Pour simplifier l'exposé, les équations de transport ci-dessous sont écrites en grandeurs instantannées vis à vis des fluctuations turbulentes, en supposant implicitement que le volume occupé par les inclusions est petit, et que ces inclusions sont petites par rapport aux échelles de la turbulence. Dans le cas d'un écoulement diphasique (injection de particules de charbon dans le plasma, par exemple), ces équations sont bien évidemment couplées aux équations de la phase dispersée (voir Simonin [1]), équations que nous n'écrirons pas ici.

2.1.1 Equations dynamiques. - L'équation de conservation de la masse présente en phase gazeuse est l'équation dite de continuité :

$$
\frac{\partial \rho}{\partial \mathrm{t}}+\operatorname{div}(\rho \mathbf{u})=\Gamma
$$

$\Gamma$ représente ici le flux de masse échangé avec les inclusions (particules, goutelettes) qui peuvent être présentes au sein de la phase gazeuse.

Le bilan de quantité de mouvement est traduit quand à lui par l'équation de Navier-Stokes :

$$
\begin{array}{r}
\frac{\partial \mathbf{u}}{\partial t}+\mathbf{u} \cdot \overline{\overline{\operatorname{grad}}}(\mathbf{u})=-\frac{1}{\rho} \operatorname{grad}(p)+\frac{1}{\rho} \operatorname{div}(\overline{\bar{T}})+ \\
+\mathrm{g}+\frac{1}{\rho} \mathbf{F},
\end{array}
$$

où $\overline{\bar{T}}$ désigne le tenseur des contraintes visqueuses; F est de son côté la force (par unité de volume) exercée par les particules sur l'ecoulement gazeux.

La masse volumique est donnée par l'equation d'état, que l'on écrit ici (par commodité de l'exposé) comme l'équation d'état du gaz parfait :

$$
p=\rho \frac{R}{M} T
$$

Dans le cas où la phase gazeuse est constituée du mélange de $n$ espèces chimiques différentes, que l'on numérotera dans la suite de $i=1 \mathrm{à} n$, la masse molaire du mélange s'exprime à partir des masses molaires $M_{i}$ et des fractions massiques $X_{i}$ des $n$ espèces :

$$
\frac{1}{M}=\sum_{i=1}^{n} \frac{X_{i}}{M_{i}}
$$

2.1.2 Bilans de masse et d'énergie en phase gazeuse. Le bilan de masse du constituant $i$ s'écrit :

$\frac{\partial X_{i}}{\partial t}+\mathbf{u} \cdot \operatorname{grad} X_{i}=\frac{1}{\rho} \operatorname{div}\left(\rho D_{i} \operatorname{grad} X_{i}\right)+Q_{i}+R_{i}$

Ici, $D_{i}$ est le coefficient de diffusion du constituant $i, Q_{i}$ est le taux d'apparition ou de disparition du constituant $i$ par suite des échanges avec les particules solides ou les goutelettes liquides transportées par l'écoulement gazeux, et $R_{i}$ le taux d'apparition ou disparition du constituant $i$ par suite des réactions chimiques.

On a supposé ici, dans un but de simplification, que le flux diffusif du constituant $i$, de fraction massique $X_{i}$, ne dépend que de gradient de $X_{i}$, ce qui est inexact. En fait, dans le cas des écoulements turbulents, l'importance des flux diffusifs est faible par rapport aux flux turbulents, ce qui minimise les conséquences de cette approximation.

Le bilan d'énergie s'écrit, de son côté :

$$
\begin{aligned}
\rho C_{\mathrm{p}}\left(\frac{\partial T}{\partial t}\right. & +\mathbf{u} \cdot \operatorname{grad} T)-\left(\frac{\partial p}{\partial t}+\mathbf{u} \cdot \operatorname{grad} p\right)= \\
& =\operatorname{div}(\lambda \operatorname{grad} T)+Q+E+S-\rho h_{i} R_{i}
\end{aligned}
$$

Ici, $Q$ est le terme d'échange d'énergie avec particules ou goutelettes, $E$ est le terme d'effet Joule lié à la présence éventuelle de l'arc électrique (nul dans le cas où l'étude ne concerne que la zone de plasma en extinction), $S$ est un terme d'échauffement ou de refroidissement par rayonnement, et le dernier terme correspond à l'énergie dégagée ou absorbée par les réactions chimiques. On a noté $h_{i}$ l'enthalpie massique du constituant $i$ qui ne dépend que de la température (et de la pression), et qui comprend l'energie de formation du corps $i, h_{i 0}$, ainsi que l'énergie stockée dans le corps $i$ sous forme sensible, c'est à dire :

$$
h_{i}(T)=h_{i 0}+\int_{0}^{T} C_{p i} \mathrm{~d} T
$$

Dans la suite, on négligera les termes de variation de pression au premier membre de (6), qui ne sont importants que lorsque le nombre de Mach est supérieur à, disons, 0,5. D'une manière générale, l'analyse faite ici se limite aux cas pour lesquels la variation relative de pression est faible.

En faisant apparaitre l'enthalpie massique totale du mélange, définie comme :

$$
H\left(T, X_{j, j=1} \text { à } n\right)=\sum_{i=1}^{n} X_{i} h_{i}(T)
$$

il est possible de faire disparaitre du bilan d'énergie le terme de réaction chimique, en écrivant l'équation suivante :

$$
\begin{array}{r}
\frac{\partial H}{\partial t}+\mathbf{u} \cdot \operatorname{grad} H=\frac{1}{\rho} \operatorname{div}\left(\frac{\lambda}{C_{\mathrm{p}}} \operatorname{grad} H\right)- \\
-\frac{1}{\rho} \operatorname{div}\left(\frac{\lambda h_{i}}{C_{\mathrm{p}}} \operatorname{grad} X_{i}\right)+ \\
+\frac{h_{i}}{\rho} \operatorname{div}\left(\rho D_{i} \operatorname{grad} X_{i}\right)+\frac{Q+E+S+Q_{i} \cdot h_{i}}{\rho}
\end{array}
$$

Au second membre, on vérifie facilement que la somme des deuxième et troisième termes est nulle si les propriétés physiques sont un peu particulières, à savoir :

- les $C_{\mathrm{p} i}$ sont constants et tous égaux entre eux, $-\lambda$ est constant, 
- les coefficients de diffusion sont égaux entre eux, avec de plus : $\left.D_{i}=D=\lambda / \rho C_{\mathrm{p}}\right)$. En fait, dans un écoulement turbulent, les termes de diffusion et de thermoconduction sont négligeables dans les équations moyennées vis à vis des flux turbulents, aussi cette hypothèse est-elle de peu de conséquences.

\subsection{LE PROBLÈME DES ECOULEMENTS TURBULENTS.} - Dans le cas des écoulements turbulents, que l'on rencontre dans pratiquement toutes les applications industrielles, les seules grandeurs accessibles au calcul sont les grandeurs moyennes.

Dans les écoulements à masse volumique variable, on utilise habituellement les moyennes de Favre, ou moyennes pondérées par la masse volumique, définies cómme suit (le symbole barre désigne ici la moyenne statistique classique) :

$$
\tilde{G}=\frac{\overline{\rho G}}{\bar{\rho}}
$$

On notera dans la suite $G^{\prime \prime}$ la fluctuation de $G$ par rapport à la moyenne de Favre définie par (9), et $G^{\prime}$ la fluctuation de $G$ par rapport à la moyenne statistique classique.

Pour obtenir des équations sur les grandeurs moyennes, il est nécessaire de moyenner les équations instantanées de bilan de masse, quantité de mouvement, énergie, et masse des différents constituants du mélange (voir paragraphe 2.4). C'est au cours de cette opération de moyennage qu'apparaissent les termes de diffusion turbulente, qui viennent souvent oblitérer l'effet des termes de diffusion moléculaire.

Ces termes de diffusion turbulente sont :

- dans (2), la divergence du tenseur de Reynolds $\overline{\bar{R}}$ de composantes $R_{i j}=\overline{\rho u_{i}^{\prime \prime} u_{j}^{\prime \prime}}$;

- dans (5), la divergence du flux turbulent de masse de $i, \Phi_{i}$, de composantes $\Phi_{i, j}=\overline{\rho X_{i}^{\prime \prime} u_{j}^{\prime \prime}}$;

- dans (8), la divergence du flux turbulent d'en-

thalpie $\Phi_{H}$, de composantes $\Phi_{H, j}=\overline{\rho H^{\prime \prime} u_{j}^{\prime \prime}}$.

Par ailleurs, tous les termes sources de $(2,5,8)$ doivent être moyennés. Ceci pose un très gros problème pour le terme de réaction chimique $R_{i}$ qui s'exprime de façon non linéaire en fonction de la température et de la concentration des différentes espèces réactives. Il serait ainsi tout à fait faux d'écrire :

$$
\tilde{R}_{i}=R_{i}\left(\tilde{T}, \tilde{X}_{j}\right)
$$

L'hypothèse d'équilibre chimique (lorsqu'elle se justifie) permet de contourner cette difficulté. Dans les cas où la prise en compte des cinétiques chimiques sera nécessaire, on recherchera à appliquer les types de méthodes actuellement développées pour la prise en compte des cinétiques dans les problèmes de combus- tion turbulente comme par exemple la méthode PEUL de Borghi et Pourbaix [2].

\subsection{UTILISATION DE L'HYPOTHĖSE D'ÉQUILIBRE CHI-} MIQUE.

2.3.1 La composition atomique du mélange est constante. - Si la composition atomique du mélange est constante (cas d'un plasma d'air injecté dans un écoulement d'air, par exemple), la composition du mélange est simplement (pour une pression donnée) une fonction de la température, $X_{j}^{\mathbf{e}}(T)$, et la température peut être déduite de l'enthalpie totale grâce à l'existence d'une loi enthalpie totale - température biunivoque, selon (7):

$$
H(T)=H\left(T, X_{j}^{\mathbf{e}}(T)\right)
$$

et

$$
\tilde{H}=H(\tilde{T})
$$

L'équation (12) résulte évidemment d'une approximation : la non-linéarité de la relation $H(T)$ est "faible". C'est avec cette hypothèse qu'ont été faits tous les calculs de chauffage de gaz par plasma à ce jour (paragraphe 3.2).

\subsubsection{La composition atomique résulte du mélange de} deux gaz. - Nous considérons ici le cas (Fig. 1) où la composition atomique du mélange réactif en un point résulte du mélange de deux espèces notées $\mathrm{A}_{1}$ et $\mathrm{A}_{2}$ : par exemple, mélange d'un plasma d'argon-hydrogène $\left(A_{1}\right)$ avec du méthane froid $\left(A_{2}\right)$. Ce cas présente une certaine similitude avec une flamme de diffusion, qui résulte du mélange d'un combustible et d'un oxydant. Nous pouvons alors nous inspirer de la démarche de modélisation des flammes de diffusion à cinétique rapide (voir [2]), en la généralisant largement pour tenir compte de la complexité des espèces présentes en milieu plasma.

Si l'on suppose que tous les coefficients de diffusion $D_{i}$ des différentes espèces entre eux, $\left(D_{i}=D\right)$ ou que l'écoulement est suffisamment turbulent pour que les termes de diffusion moléculaire soient négligeables, alors la composition atomique en chaque point peut être caractérisée par une grandeur unique $f$, appelée taux de mélange. Ce taux de mélange est défini à partir des fractions massiques en éléments $y_{j}$ (ex. C, H, etc...), conventionnellement par :

$$
f=\frac{y_{j}-y_{j 2}}{y_{j 1}-y_{j 2}}
$$

avec: $-f=1$ dans $\mathrm{A}_{1}$,

$$
-f=0 \text { dans } \mathrm{A}_{2} \text {. }
$$

$y_{j 1}$ et $y_{j 2}$ sont les fractions massiques de chaque élément $j$ pour les deux gaz injectés $\mathbf{A}_{1}$ et $\mathbf{A}_{2}$. 

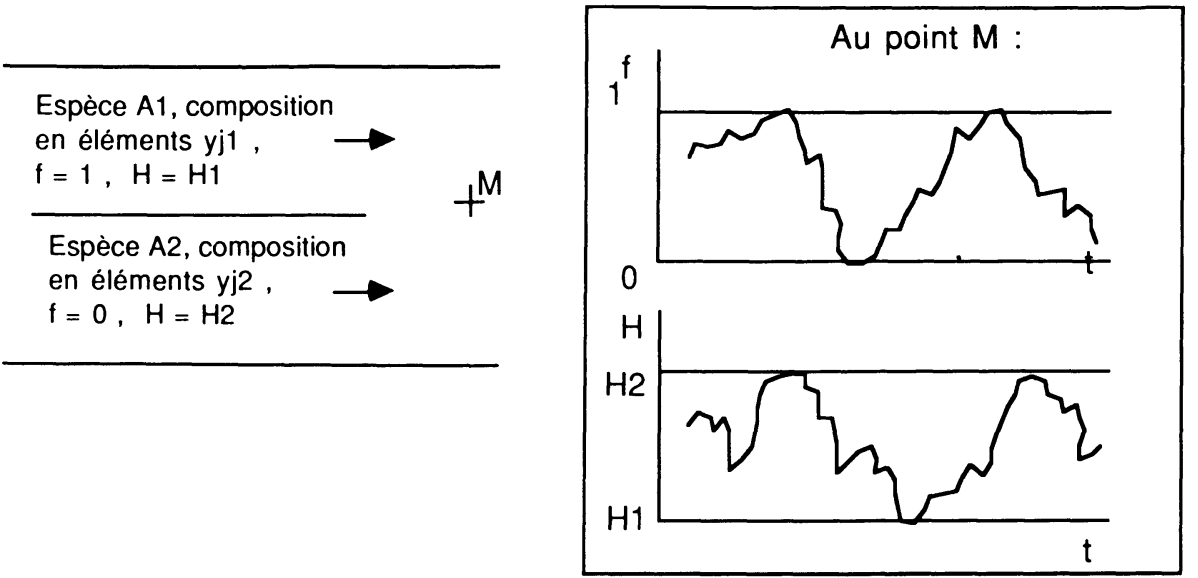

Fig. 1. - Croquis de définition : flamme de diffusion ou mélange de deux gaz plasmagènes $A_{1}$ et $A_{2}$. [Definition sketch : diffusion flame or mixing of the plasma of two gases $A_{1}$ and $A_{2}$.]

$f$ est solution de l'équation de transport suivante :

$$
\frac{\partial f}{\partial t}+\mathbf{u} \cdot \operatorname{grad} f=\frac{1}{\rho} \operatorname{div}(\rho D \operatorname{grad} f)+Q_{f}
$$

$Q_{f}$ provient de l'échange de masse avec les particules ou les gouttes. Cette équation n'a pas d'autre terme source car, bien sûr, les fractions massiques locales en éléments ne sont pas directement affectées par les réactions chimiques.

Remarquons par ailleurs que toute fonctionnelle $Y(f)$ de $f$ seul peut être moyennée si la fonction de densité de probabilité de $f, P(f)$, est connue :

$$
\widetilde{Y(f)}=\int_{0}^{1} Y(f) P(f) d f
$$

La forme de cette fonction de densité de probabilité est en pratique (voir [2]) présumée sous la forme d'une fonction bêta (d'utilisation classique en statistiques), a partir de la donnée en chaque point de la moyenne et de la variance :

$$
\tilde{f} \text { et } \widetilde{f^{\prime \prime 2}} \text {, }
$$

qui sont chacune solution d'une équation de transport (Eqs. (14 bis) et (27), au paragraphe 2.4).

Revenons à notre mélange de gaz plasmagènes $A_{1}$ et $\mathrm{A}_{2}$. La fraction massique en éléments du mélange correspondant à une certaine valeur de $f$ est caractérisée, pour chaque élément $j$, par : $y_{j}=(1-f) y_{j 2}+f y_{j 1}$, d'après (13). On pourrait envisager de calculer l'équilibre chimique du mélange caractérisé par les $y_{j}$, et d'en déduire la température à partir de l'enthalpie locale moyenne, en écrivant :

$$
\tilde{T}=T^{\mathrm{e}}(\tilde{H}, \tilde{f}) \quad(\text { faux }),
$$

où $T^{\mathrm{e}}(H, f)$ est l'inverse de la fonctionnelle $H^{\mathrm{e}}(T, f)$ donnée par les calculs d'équilibre chimique effectués pour différentes compositions atomique du mélange (compositions caractérisées par $f$ ). Cette approximation reviendrait, compte tenu de la non-linéarité de $T^{\mathbf{e}}(H, f)$, à négliger l'influence de la turbulence sur les taux de réaction (voir Eq. (10 faux) au paragraphe 2.2), ce qui, on le sait par l'analyse des problèmes de combustion, conduit à des températures moyennes de flamme beaucoup trop élevées et à des zones de réaction trop minces. On propose dans la suite une méthode approchée pour résoudre cette difficulté.

Le calcul exact de la temperature moyenne à partir des données d'équilibre $T^{\mathbf{e}}(H, f)$ s'écrit :

$$
\tilde{T}=\int_{0}^{1} \int_{H_{1}}^{H_{2}} T^{\mathrm{e}}(H, f) P(H, f) \mathrm{d} H \mathrm{~d} f
$$

où $P(H, f)$ est la densité de probabilité conjointe de $H$ et de $f$.

On ne dispose, en chaque point, que des valeurs moyennes :

$\tilde{H}$ et $\tilde{f}$, solutions des équations de transport correspondantes, ainsi que de la fonction de densité de probabilité $P(f)$, que l'on a déjà introduite plus haut.

Dans le cas où les équations et les conditions aux limites pour l'enthalpie $H$ sont de la même forme que pour le taux de mélange $f$, en l'absence de termes sources (radiatif en particulier) dans le bilan énergétique, et pour des propriétés physiques un peu idéales (celles pour laquelle l'equation (3) se simplifie), on a :

$$
f=\frac{H-H_{2}}{H_{1}-H_{2}}
$$

On formule alors l'hypothèse suivante, qui est une généralisation de ce résultat particulier.

Hypothèse : les fluctuations de $H$ sont corrélées uni- 
quement aux fluctuations de $f$, par une fonctionnelle $H(f)$, définie par :

a) $H(0)=H_{1}$ et $H(1)=H_{2}$ (réalisabilité) ;

b) $\quad \tilde{H}=\int_{0}^{1} H(f) P(f) \mathrm{d} f$

où la moyenne de $H$ est solution de l'équation de transport (8bis), avec termes sources s'il y a lieu. Cette fonctionnelle $H(f)$ pourrait être approchée par une équation du second degré.

Avec cette hypothèse, $P(H, f)$ se ramène simplement à $P(f)$, car la probabilite de rencontrer un couple $(H, f)$ qui ne soit pas sur la courbe $H(f)$ est nulle.

$P(H, f)=\delta(H(f)) P(f)$, et on peut alors écrire :

$$
\tilde{T}=\int_{0}^{1} T^{\mathrm{e}}(H(f), f) P(f) \mathrm{d} f
$$

Numériquement, les calculs seront évidemment un peu lourds, les fonctions $T^{\mathbf{e}}(H, f)$ étant nécessairement tabulées à partir des résultats des calculs d'équilibre chimique (qui donneront $H^{\mathrm{e}}(T)$ pour un certain nombre de valeurs de $f$ ).

La composition détaillée du mélange en un point donné peut être calculée de la même façon : si on note par exemple $X_{\boldsymbol{H}}=X_{\boldsymbol{H}^{\mathrm{e}}}(T, f)$ la fraction massique en radical $H$ calculée à l'équilibre, on calculera la fraction massique moyenne en radical $H$ par :

$$
\widetilde{X_{H}}=\int_{0}^{1} X_{H}^{\mathrm{e}}\left(T^{\mathrm{e}}\{H(f), f\}, f\right) P(f) \mathrm{d} f
$$

Pour illustrer l'utilisation de cette méthode, le calcul d'une flamme de diffusion turbulente est présenté au paragraphe 3.3 .

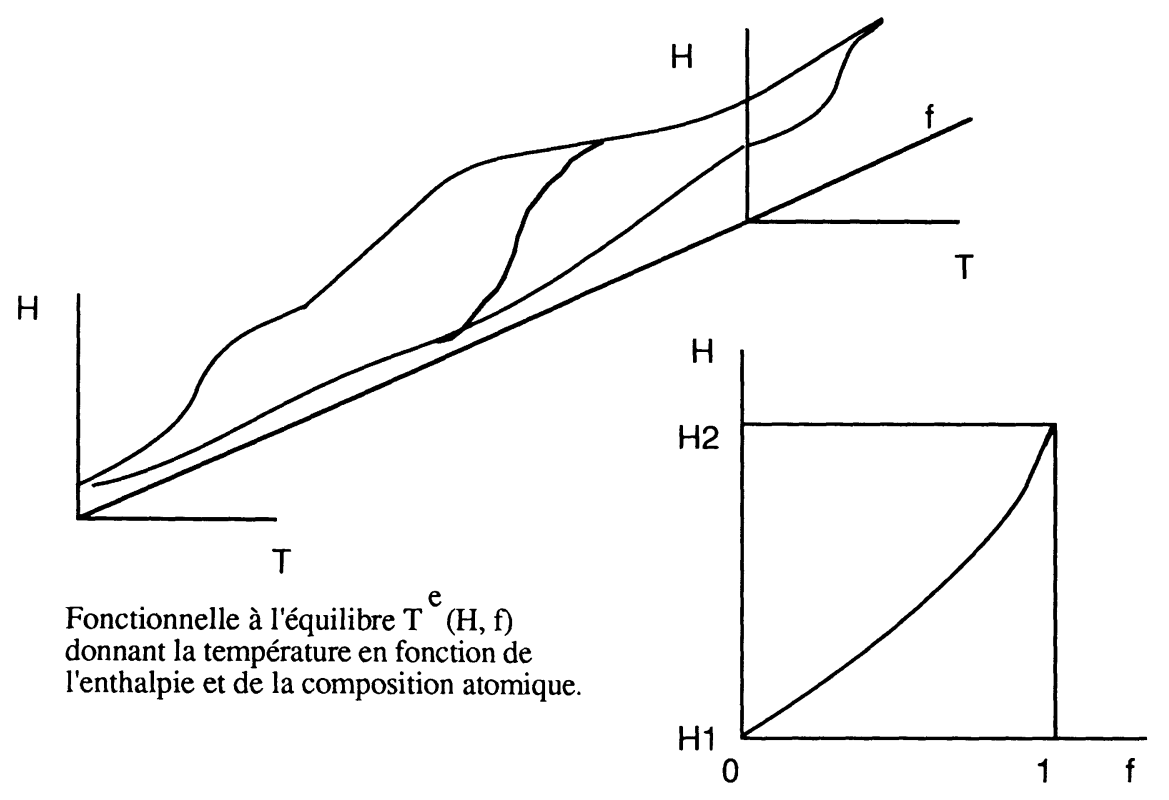

Fonctionnelle reliant, par hypothèse, les fluctuations turbulentes de $\mathrm{H}$ à celles de $\mathrm{f}$.

Fig. 2. - Croquis de définition, relatif aux équations (16) à (20).

[Definition sketch related to equations (16) to (20).]

2.4 LE MODELLE DE TURBULENCE. - Les équations moyennées qu'il faut finalement résoudre sont les suivantes, pour un problème stationnaire "en moyenne" :

$$
\operatorname{div}(\rho \tilde{\mathbf{u}})=\bar{\Gamma}
$$

$\bar{\rho} \tilde{\mathbf{u}} \cdot \overline{\overline{\operatorname{grad}}}(\tilde{\mathbf{u}})=-\operatorname{grad}(\bar{p})+\operatorname{div} \overline{\overline{(\overline{\mathrm{T}}-R)}}+\bar{\rho} \mathbf{g}+\overline{\mathbf{F}}$

$$
\bar{p}=\bar{\rho} R \tilde{T} \sum_{i=1}^{n} \frac{\tilde{X}_{i}}{M_{i}}
$$

$\bar{\rho} \tilde{\mathbf{u}} \cdot \operatorname{grad} \tilde{X}_{i}=\operatorname{div}\left(\overline{\rho D} \operatorname{grad} \tilde{X}_{i}-\boldsymbol{\Phi}_{i}\right)+\bar{\rho} \tilde{Q}_{i}+\bar{\rho} \tilde{R}_{i}$

$\bar{\rho} \tilde{\mathbf{u}} \cdot \operatorname{grad} \tilde{H}=\operatorname{div}\left(\frac{\lambda}{C_{\mathrm{p}}} \operatorname{grad} \tilde{H}-\boldsymbol{\Phi}_{H}\right)+$ 


$$
\bar{\rho} \tilde{\mathbf{u}} \cdot \operatorname{grad} \tilde{f}=\operatorname{div}\left(\overline{\rho D} \operatorname{grad} \tilde{f}-\Phi_{f}\right)+\bar{\rho} \tilde{Q}_{f}
$$

Dans (14 bis), $\Phi_{f}$ est un flux turbulent de même nature que $\Phi_{i}$ ou $\Phi_{H}$. L'écriture de ces équations moyennées ne va pas, remarquons-le, sans poser quelques problèmes : ainsi, on a supposé en écrivant (3 bis) que la masse molaire du mélange est non fluctuante (ce qui est inexact), et l'ecriture moyennee des termes de diffusion moléculaire dans ( 8 bis), ( 5 bis), (14 bis) n'est pas très rigoureuse (mais de toute façon leur formulation même dans les équations instantanées n'est pas très exacte).

La modélisation la plus simple des transferts turbulents utilise le concept de viscosité turbulente pour exprimer les tensions de Reynolds et les flux turbulents de masse et d'énergie :

$$
\begin{array}{r}
R_{i j}=\overline{\rho u_{i}^{\prime \prime} u_{j}^{\prime \prime}}=-\mu_{\mathrm{T}}\left(\frac{\partial \tilde{u}_{i}}{\partial x_{j}}+\frac{\partial \tilde{u}_{j}}{\partial x_{i}}\right)+ \\
+\frac{2}{3} \delta_{i j}\left(\bar{\rho} k+\mu_{\mathrm{T}} \operatorname{div}(\tilde{\mathbf{u}})\right) \\
\Phi_{i, j}=\overline{\rho X_{i}^{\prime \prime} u_{j}^{\prime \prime}}=-\kappa_{\mathrm{T}} \frac{\partial \tilde{X}_{i}}{\partial x_{j}} \\
\Phi_{H, j}=\overline{\rho H^{\prime \prime} u_{j}^{\prime \prime}}=-\kappa_{\mathrm{T}} \frac{\partial \tilde{H}_{i}}{\partial x_{j}}
\end{array}
$$

Pour $\Phi_{f}$, on adopte une modélisation identique à (23). L'égalité des coefficients de diffusion dans (22) et (23) résulte de l'analogie entre les transferts turbulents de masse et d'énergie.

La viscosité turbulente $\mu_{\mathrm{T}}$ et la diffusivité turbulente $\kappa_{\mathrm{T}}$ sont donnés par le modèle à deux équations de transport, dit modele $k-\varepsilon$ :

$$
\mu_{T}=\bar{\rho} C_{\mu} \frac{k^{2}}{\varepsilon}, \quad \text { et } \quad \kappa_{\mathrm{T}}=\frac{\mu_{\mathrm{T}}}{\sigma_{\mathrm{T}}}
$$

A masse volumique variable, les équations de transport de l'énergie turbulente $\mathbf{k}$ et de sa dissipation $\varepsilon$ s'écrivent (toujours pour un problème stationnaire "en moyenne") :

(a) Energie turbulente :

$$
\bar{\rho} \tilde{\mathbf{u}} \cdot \operatorname{grad} k=\operatorname{div}\left(\frac{\mu_{\mathrm{T}}}{\sigma_{\mathbf{k}}} \operatorname{grad} k\right)+P-\bar{\rho} \varepsilon+\Pi_{k},
$$

où le terme de production s'écrit : $P=-\overline{\rho u_{i}^{\prime \prime} u_{j}^{\prime \prime}} \frac{\partial \tilde{u}_{i}}{\partial x_{j}}+$ $\overline{\frac{\rho^{\prime} u_{i}^{\prime}}{\bar{\rho}}} \frac{\partial \bar{p}}{\partial x_{i}}$.

Dans ce dernier terme, $\overline{\rho^{\prime} u_{i}^{\prime}}$ est modélisé comme : $-\frac{\kappa_{\mathrm{T}}}{\bar{\rho}} \frac{\partial \bar{\rho}}{\partial x_{i}}$ (b) Dissipation :

$$
\begin{array}{r}
\bar{\rho} \tilde{\mathbf{u}} \cdot \operatorname{grad} \varepsilon=\operatorname{div}\left(\frac{\mu_{\mathrm{T}}}{\sigma_{\varepsilon}} \operatorname{grad} \varepsilon\right)+ \\
+\frac{\varepsilon}{k}\left(\mathbf{c}_{\varepsilon 1} P-C_{\varepsilon 2} \bar{\rho} \varepsilon\right)+\Pi_{\varepsilon} .
\end{array}
$$

(c) Variance des fluctuations de $\mathrm{f}$ :

$\bar{\rho} \tilde{\mathbf{u}} \cdot \operatorname{grad} \widetilde{f^{\prime \prime 2}}+\operatorname{div}\left(\kappa_{\mathrm{T}} \operatorname{grad} \widetilde{f^{\prime \prime 2}}\right)+P_{f}-\bar{\rho} \varepsilon_{f}+\Pi_{f}$.

La "production" est ici : $P_{f}=-2 \overline{\rho u_{j}^{\prime \prime} f^{\prime \prime}} \frac{\partial \tilde{f}}{\partial x_{j}}$, et la dissipation est modélisée comme : $\varepsilon_{f}=\frac{1}{R_{f}} \varepsilon \frac{\overline{f^{\prime 2}}}{k}$.

$\Pi_{k} \Pi_{\varepsilon^{\prime}} \Pi_{f}$ résultent de l'intéraction avec les inclusions et ne sont pas analysés ici (voir [1]).

Ce modele $k-\varepsilon$ (Launder et Spalding [4]), complété de l'équation de transport de la variance des fluctuations de $f$, a été très largement utilisé pour le calcul des écoulements incompressibles. Tous les calculs présentés au prochain paragraphe ont été effectués en utilisant ce modele, avec un jeu tout à fait standard des constantes (sauf mention contraire). Les résultats sont cependant parfois améliorés en utilisant des valeurs "non standard" du nombre de Prandtl (ou de Schmidt) turbulent $\sigma_{\mathrm{T}}$, ce qui définit les limites de ce modele $k$ - $\varepsilon$.

\begin{tabular}{|ccccccc|}
\hline$C_{\mu}$ & $\sigma_{T}$ & $C_{\varepsilon 1}$ & $C_{\varepsilon 2}$ & $\sigma_{k}$ & $\sigma_{\varepsilon}$ & $R_{f}$ \\
\hline 0.09 & 1 & 1.44 & 1.92 & 1 & 1.3 & 0.8 \\
\hline
\end{tabular}

Depuis quelques années, des méthodes numériques précises de résolution des équations de transport des tensions de Reynolds et des flux turbulents pour des écoulements elliptiques (avec recirculations potentielles) ont vu le jour : pour ce faire, de très importantes difficultés d'ordre numériques ont dû être résolues (difficultés d'autant plus importantes que l'on est plus exigeant quand à la minimisation de la diffusion numérique). Ces travaux ont été réalisés avec des algorithmes :

- de type Teach, à UMIST [5] ;

- de type Mac Cormack implicite [6], dans la direction principalement des écoulements à grand nombre de Mach ;

- à pas fractionnaires comme ceux utilisés dans les présents calculs (Kanniche, Baron, Viollet [7]).

La résolution complète des équations de transport des tensions de Reynolds et des flux turbulents sera probablement largement utilisée à l'avenir dans le calcul d'écoulements plasma.

2.5 MÉTHODES NUMÉRIQUES ET PRISE EN COMPTE DE GÉOMÉTRIES TRIDIMENSIONNELLES COMPLEXES. La résolution numérique des équations ci-dessus est effectuée en utilisant des méthodes à pas fraction- 
naires, avec un algorithme instationnaire modifié par une technique de pas de temps local qui permet d'accélérer la convergence vers les solutions stationnaires. La résolution des équations comprend ainsi trois étapes (voir [8]):

(a) étape de convection, où l'on utilise une méthode de caractéristiques (voir [9]) dans le but de minimiser la diffusion numérique ; cette diffusion numérique résulte d'une mauvaise discrétisation des termes non linéaires de convection, et, s'ajoutant en quelque sorte aux phénomènes de transport que l'on modélise, peut venir altérer notablement la qualité des résultats.

(b) étape de diffusion et d'application des termes sources ;

(c) étape de calcul de la pression, par une méthode de volumes finis, et de correction du champ de vitesse pour satisfaire à la conservation de la masse.

L'utilisation d'un maillage structuré pour représenter des écoulements en conduites (cas très fréquent de l'accouplement d'une torche à plasma sur une conduite industrielle) est rendue possible par :

- l'utilisation de mailles de pas variable en espace, et de conditions aux limites sur des frontières "obliques" par rapport à la maille ;

- l'utilisation de mailles curvilignes pour suivre les coudes d'une conduite.

De nombreuses validations de cet outil de calcul (système Estet-Perséphone) ont été effectuées pour des écoulements isothermes ou présentant de faibles variations de masse volumique. On a en particulier vérifié, en écoulement isotherme, que le calcul prédit correctement les circulations secondaires dans une conduite coudé (Gabillard et Viollet [10]).

Le développement récent de méthodes de calcul tridimentionnelles en éléments finis permet d'envisager pour le futur l'utilisation de ces méthodes aux calculs des écoulements des plasmas thermiques : ces méthodes permettront une prise en compte plus facile des géométries compliquées qui sont souvent rencontrées dans l'étude de procédés industriels.

\section{Démarche de validation.}

\subsection{ETUDE D'ÉCOULEMENTS NON RÉACTIFS À MASSE VOLUMIQUE VARIABLE.}

3.1.1 Situation du sujet. - Des tentatives ont été faites il y a quelques années au LNH pour aménager le modele $k-\varepsilon$ en tenant compte des fluctuations de masse volumique (Viollet [11]). La plupart des travaux de modélisation plus récents ont été cependant effectués en utilisant des fermetures de corrélations du second ordre. Citons en particulier :

- les travaux récents de Chassaing et Herrard [12,13], effectués en apportant une attention particulière à la modélisation des corrélations pression-déformation (on utilise des moyennes de Reynolds), appliqués au calcul d'un jet par une méthode parabolique ;

- des résultats présentés au colloque Euromech $237[14,15]$ qui montrent que des résultats très encourageants peuvent être obtenus dans des écoulements elliptiques par une fermeture du second ordre formulée en moyennes de Favre, en introduisant seulement les termes de corrélation densité-gradient de pression qui apparaissent naturellement dans l'écriture des équations moyennées.

3.1.2 Etude d'une couche de mélange plane à masse volumique variable. - Un banc d'essai ("Echo") de mélanges de gaz hélium- $\mathrm{CO}_{2}$ a été installé au $\mathrm{LNH}$, et des mesures sur un écoulement plan (couche de mélange) sont effectuées, sur la même géométrie qu'une expérience de combustion prémélangée réalisée à l'ONERA par Moreau [16] (voir Figure 3). Les résultats correspondant à un rapport de densités $\mathbb{R}=\mathbf{4 . 2}$ sont portés figure 4 : ils montrent que le mélange est correctement prédit, et que les résultats sont meilleurs si une valeur du nombre de Schmidt turbulent plus basse que les valeurs standards ( 0.5 au lieu de 0.7 ou 1) est utilisée.

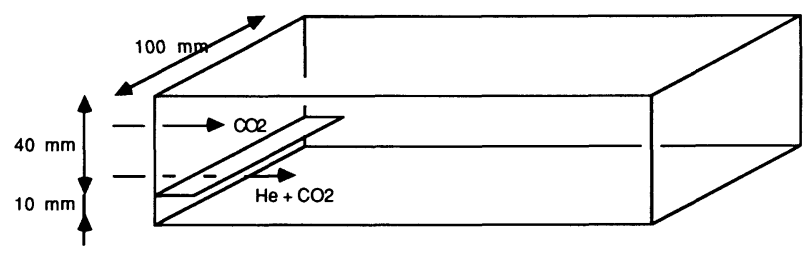

Fig. 3. - Croquis de définition : couche de mélange à densité variable.

[Definition sketch : variable density mixing layer.]

3.1.3 Etude d'une configuration tridimensionnelle. La configuration géométrique tridimensionnelle étudiée reproduit de manière un peu schématique un type de configuration habituel dans les applications industrielles des plasmas (préchauffage de l'air dans une tuyère de haut fourneau). Elle est représentée figure 5, et a fait l'objet d'une étude expérimentale sur la même installation "Echo" que pour le cas précédent (voir [19]). Le mélange $\mathrm{He}+\mathrm{CO}_{2}$ est émis ici par une buse épaisse faisant saillie à l'intérieur de la conduite, avec un angle d'incidence de $45^{\circ}$. Les comparaisons des résultats de calcul et des résultats expérimentaux pour les deux profils 2 et 3 repérés figure 5 sont portées figure 6 : les résultats de la comparaison sont très bons dans la section 2, ils se dégradent au point d'impact du jet sur la paroi.

3.2 Configurations DE MÉlange Plasma-COURANT DE DILUTION À COMPOSITION ATOMIQUE CONSTANTE. - Les calculs présentés ici concernent le mé- 


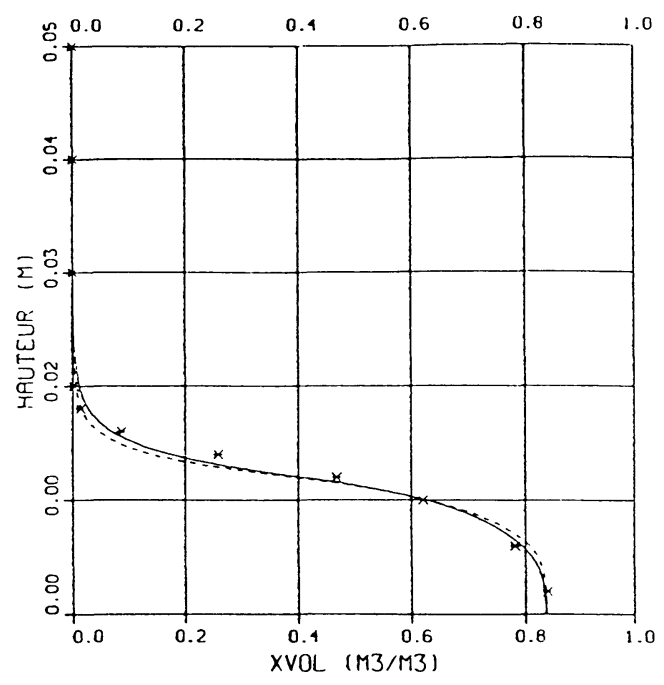

$x=0.06 \mathrm{~m}$

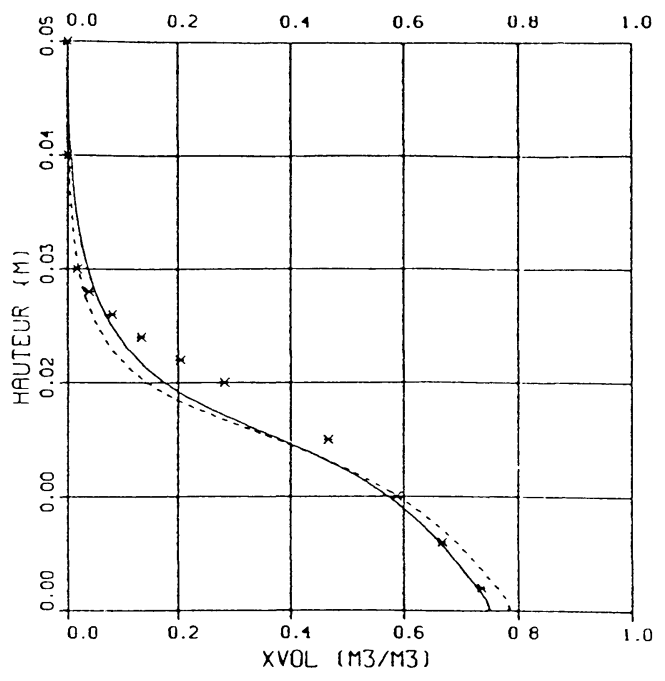

$x=0.22 \mathrm{~m}$

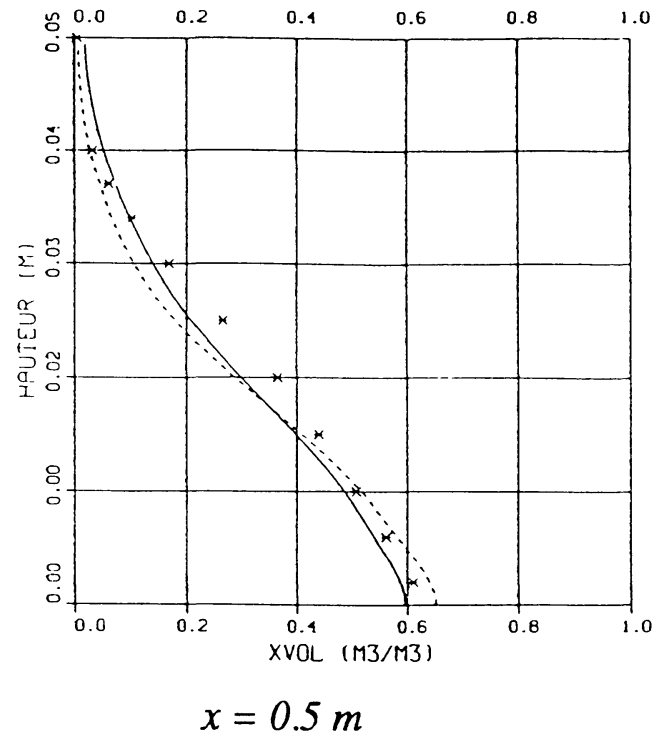

Fig. 4. - Couche de mélange à densité variable : comparaison de profils de concentration volumique en He mesurés et calculés pour deux valeurs du nombre de Schmidt turbulent. (Gabillard et Flamain [17]), à trois sections différentes en aval. Les vitesses des couches supérieure et inférieure sont resp. $3,75 \mathrm{~m} / \mathrm{s}$ et 7,6 m/s. Rapport des densités : $\mathbb{R}=4,2$. (***) mesures, $(-)$ calcul, $\sigma_{\mathrm{T}}=0,5,(-\cdots)$ calcul, $\sigma_{\mathrm{T}}=0,67$.

[Variable density mixing layer : comparison He volumic concentrations measured and computed for two values of the turbulent Schmidt number (Gabillard and Flamain [17]) at three downstream sections. The velocity of the upper and lower layers are respectively : $3.75 \mathrm{~m} / \mathrm{s}$ and $7.6 \mathrm{~m} / \mathrm{s}$. The density ratio is $\mathbb{R}=4.2$.]

lange d'un courant de dilution avec un plasma en extinction d'un même gaz. Ces calculs utilisent l'hypothèse d'équilibre avec l'approximation (12). Compte tenu des gaz plasmagènes utilisés, le rayonnement au sein du gaz est négligé.

3.2.1 Configurations axisymmétriques. - La première configuration (Fig. 7) reproduit des essais effectués en 1983 à ISSAC par l'Aérospatiale, avec une torche Aérospatiale de 1,4 MW dont le jet (plasma d'air) est émis dans un convergent en tôle mince, avec un écoulement d'air froid périphérique, prolongé par un tube cylindrique de $0,18 \mathrm{~m}$ de diamètre. Dans le calcul, la température de sortie torche est supposée constante, égale à $4000 \mathrm{~K}$, et le profil de vitesse uniforme. Les températures sont mesurées sur l'axe de symétrie loin de la torche (à 2,35 m, environ, distance à laquelle le mélange est réalisé : la température sur l'axe dans l'expérience comme dans le calcul, n'est plus que de $1130 \mathrm{~K}$ ), ainsi que sur la paroi de la tôle. Dans le calcul, cette température de paroi est calculée comme résultant de l'equilibre entre l'échange par convection à 


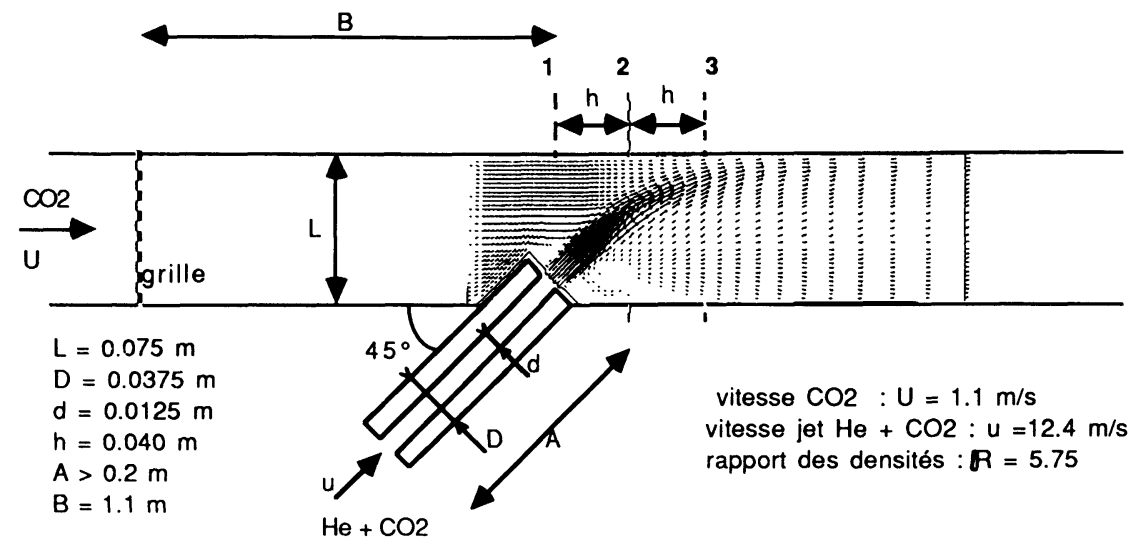

Fig. 5. - Croquis de définition de la configuration tridimensionnelle.

[Définition sketch of the three-dimensional configuration.]

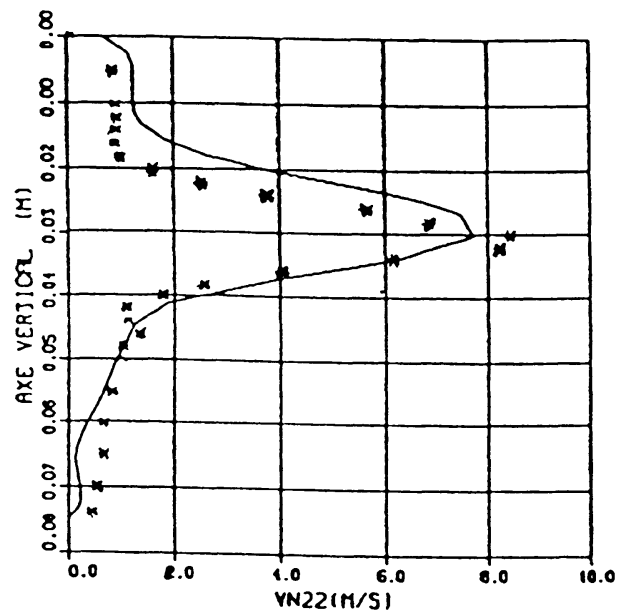

norme 2 de vilesse profil 2

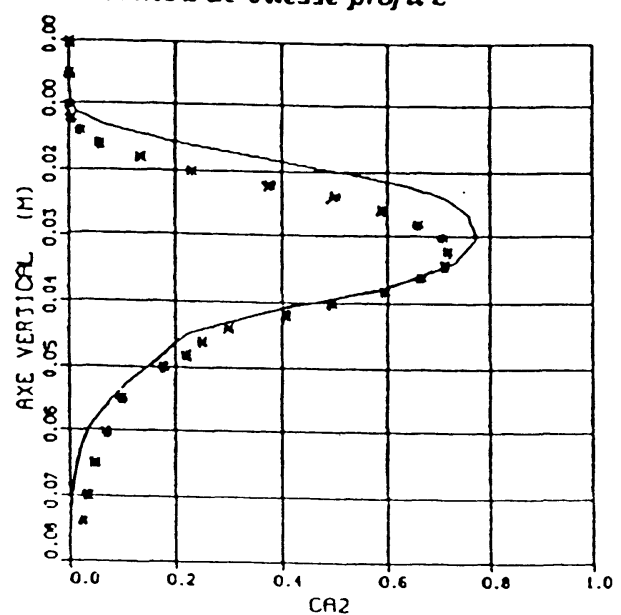

concentralion vol. adim. profil 2

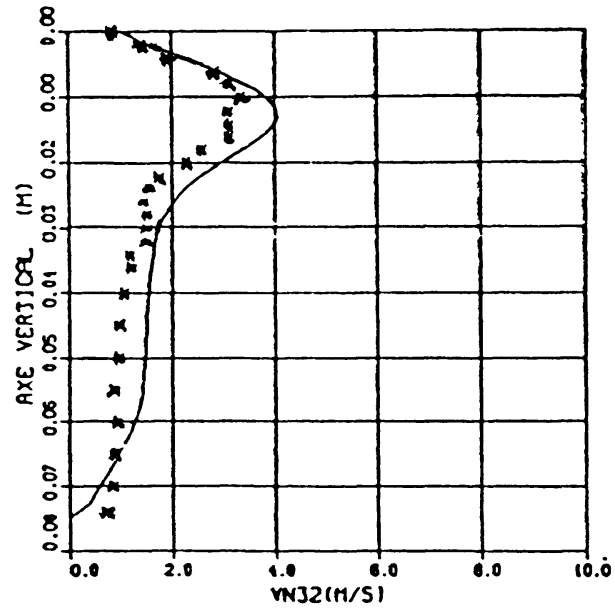

norme 2 de vilesse profil 3

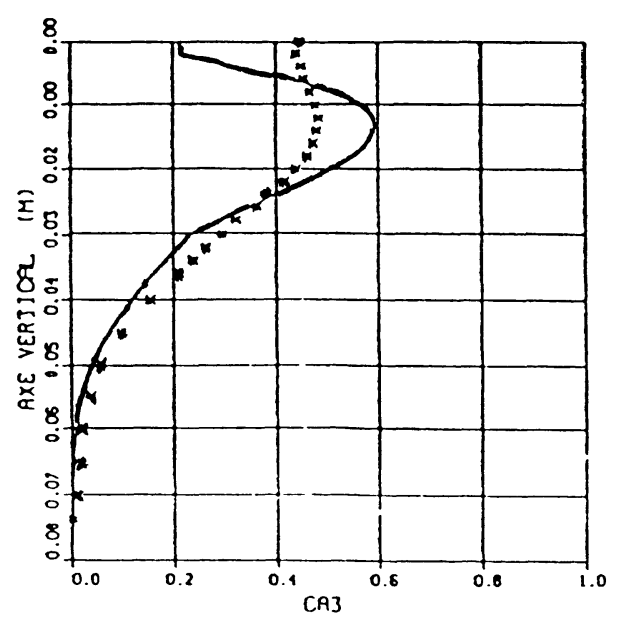

concentration vol. adim. profil 3

Fig. 6. - Profils de vitesse (en haut) et de concentration (en bas) moyennes mesurés et calculés dans les sections 2 et 3. $(-)$ calculs avec $\sigma_{\mathrm{T}}=1$ (Gabillard et Durante [18]), $(* * * * * * *)$ points expérimentaux (Viollet, Flamain, Lana, Olivier [19]).

[Profiles of mean velocity (up) and concentration (down) measured and computed in sections 2 and 3.] 
l'intérieur du tube, et le refroidissement du tube d'acier par rayonnement et convection naturelle vers le local d'essai. La figure 7 montre le maillage de calcul utilisé, le champ de vitesse calculé, ainsi que la comparaison des températures de paroi calculées et mesurées. Dans le calcul, l'effet de surchauffe locale en début de partie cylindrique du tube est sous-estimé.

La deuxième configuration reproduit une géométrie analogue à plus petite échelle, avec une torche à plasma SFEC de $10 \mathrm{~kW}$, en argon. Les expériences et les calculs ont été réalisés par Izarra [21], au GREMI pour les expériences, et à EDF-LNH pour les calculs.
Compte-tenu du caractère peu turbulent de l'écoulement en sortie de la torche, on tient compte dans le calcul de profils de température et vitesse paraboliques en sortie torche. Le mélange très rapide à l'intérieur du dispositif est bien reproduit par le calcul (Fig. 8), avec des températures sur l'axe qui se comparent bien aux résultats expérimentaux, mais les températures au voisinage de la paroi sont, dans le calcul, un peu trop basses. Dans l'ensemble des cas étudiés par Izarra, l'écart calcul-mesure ne dépasse pas $300 \mathrm{~K}$, ce qui peut paraître important, mais doit être comparé à l'écart de température important entre le plasma et le courant de dilution.

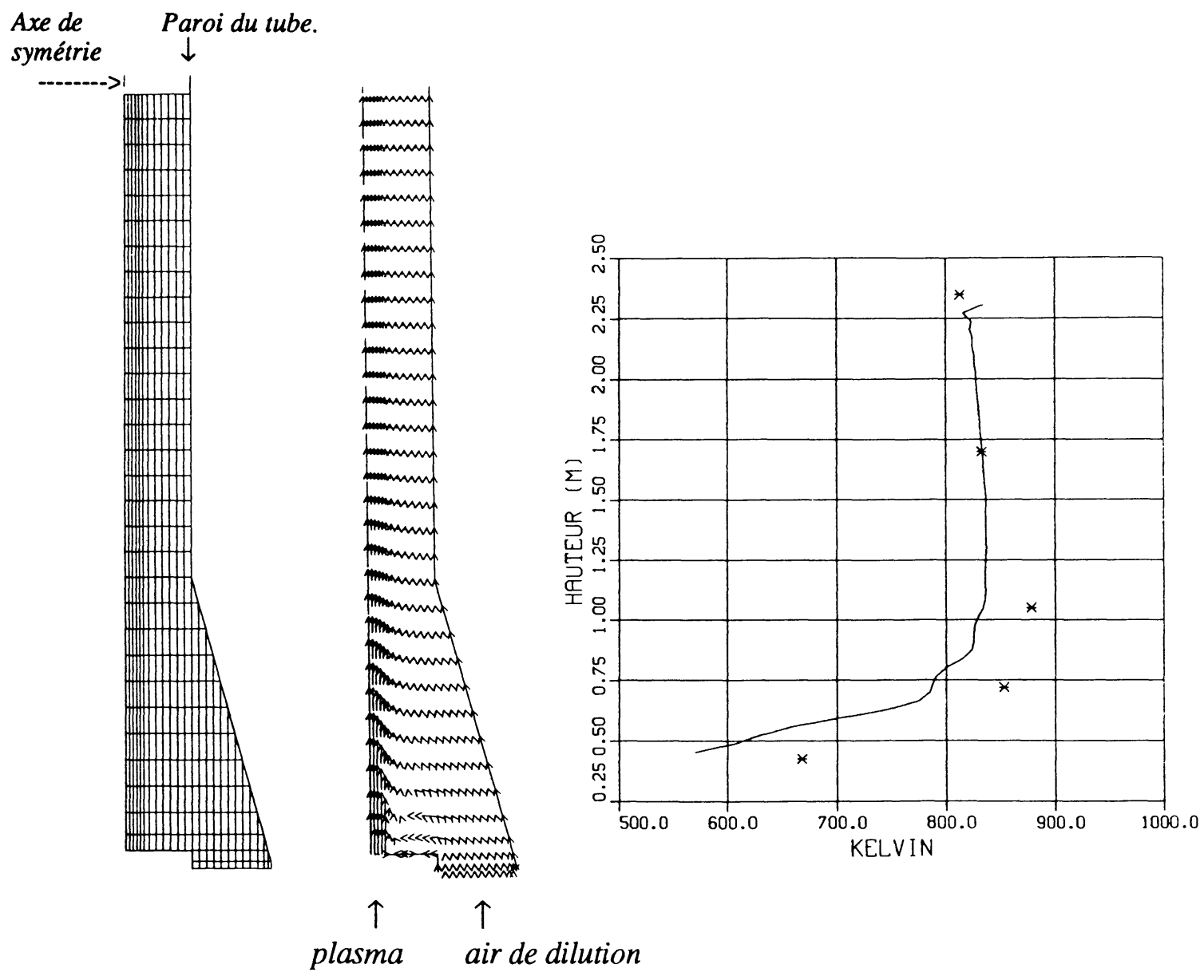

Fig. 7. - Torche à plasma Aérospatiale dans un tube convergent. De gauche à droite : maillage de calcul et géométrie ; champ de vitesse calcule ; temperatures de paroi calculees et mesurées sur la partie cylindrique du tube $\mathrm{AB}$ (le calcul figure en trait plein) - d'après Simonin et Béchart [20]. Les débits de plasma et d'air de dilution sont respectivement : $0,165 \mathrm{~kg} / \mathrm{s} \mathrm{et}$ $1,15 \mathrm{~kg} / \mathrm{s}$. Le diamètre du tube cylindrique est de $0.183 \mathrm{~m}$.

[The Aerospatiale plasma torch in a converging tube. From left to right : geometry and computing grid ; computed velocity field ; computed and measured wall temperature on the cylindrical part $\mathrm{AB}$ (the computation appears as a solid line) - from Simonin et Béchard [20]. The plasma and dilution flow rates are resp. : $0.165 \mathrm{~kg} / \mathrm{s}$ and $1.15 \mathrm{~kg} / \mathrm{s}$. The cylindrical tube diameter is $0.183 \mathrm{~m}$.] 

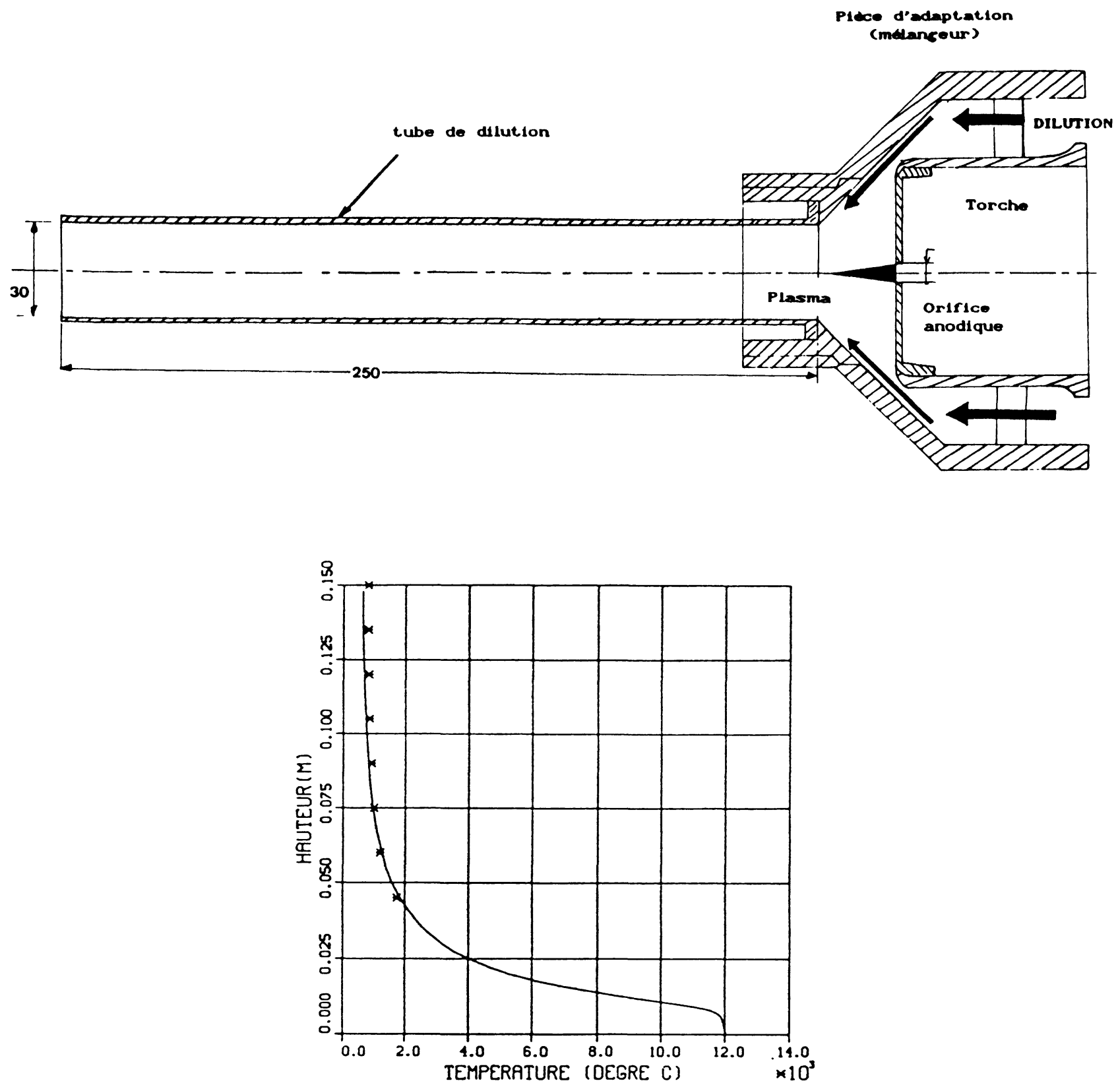

Fig. 8. - Plasma d'argon dans un écoulement d'argon annulaire (d'après Izarra [21]). Géométrie de l'expérience et profil de température sur l'axe. Débits de gaz plasmagène et du courant de dilution : respectivement $0,6 \mathrm{~g} / \mathrm{s} \mathrm{et} 6,67 \mathrm{~g} / \mathrm{s}$. $(-)$ Calcul. $(* * *)$ Mesures.

[Argon plasma discharged in an annular flow of argon (from Izarra [21]). Geometry of the experiment and axial mean temperature. The flow rates of plasma and dilution gas are respectively $0.6 \mathrm{~g} / \mathrm{s}$ and $6.67 \mathrm{~g} / \mathrm{s}$.]

3.2.2 Configuration tridimensionnelle. - Une expérimentation à l'échelle industrielle a été effectuée au laboratoire plasma des Renardières (EDF). Sa géométrie rappelle celle de la figure 5 , avec un torche à plasma incliné à $45^{\circ}$ par rapport à la conduite (de diametre aval $0,28 \mathrm{~m}$ ), avec une géométrie d'injection toutefois un peu plus complexe. La torche utilisée est une torche Aérospatiale de $2 \mathrm{MW}$.
Pour le cas qui est présenté, le rapport des débits massiques courant de dilution/plasma est de 6.5, et la torche fonctionne à une puissance de 1.1 MW. Dans la section (a), des mesures de vitesse ont été effectuées par ADL par l'ONERA, et des mesures de température ont été effectuées par DRASC (81 points de mesure) par une équipe EDF (voir [22-23]). 
Les conditions de calcul sont les suivantes : débits d'air froid et de plasma, respectivement $0,680 \mathrm{~kg} / \mathrm{s}$ et $0,110 \mathrm{~kg} / \mathrm{s}$ (soit un rapport des débits masse de 6,2); les températures d'injection du plasma et de l'air froid sont prises à $4000 \mathrm{~K}$ et $300 \mathrm{~K}$, respectivement.

La figure 9 montre la géométrie du problème, avec des champs de vitesse et température calculés. Une vue dans l'espace du maillage de calcul est portée figure 12. Dans le présent calcul, les parois de la conduite ont été supposées adiabatiques, alors que dans l'expérience ces parois sont refroidies par circulation d'eau : les champs de température ne sont donc pas directement comparables. Ces derniers (Fig. 10) montrent ce- pendant, dans le calcul comme dans l'expérience, que le jet issu de la torche a impacté la paroi opposée à cette dernière, et que le mélange est déjà largement réalisé à une distance de $0.5 \mathrm{~m}$ en aval de la torche, avec des températures maximales comparables entre le calcul et l'expérience $(1200 \mathrm{~K}$ et $1100 \mathrm{~K}$, environ). Les transferts thermiques gaz-paroi (non reproduits dans le présent calcul) ont pour conséquence de rendre l'homogénéisation de la température plus rapide. L'assymétrie observée sur les mesures de température peut être due à l'existence d'un coude en amont (non représenté dans le calcul). Les profits de vitesse calculés et mesurés (Fig. 11), se comparent de leur côté assez bien.

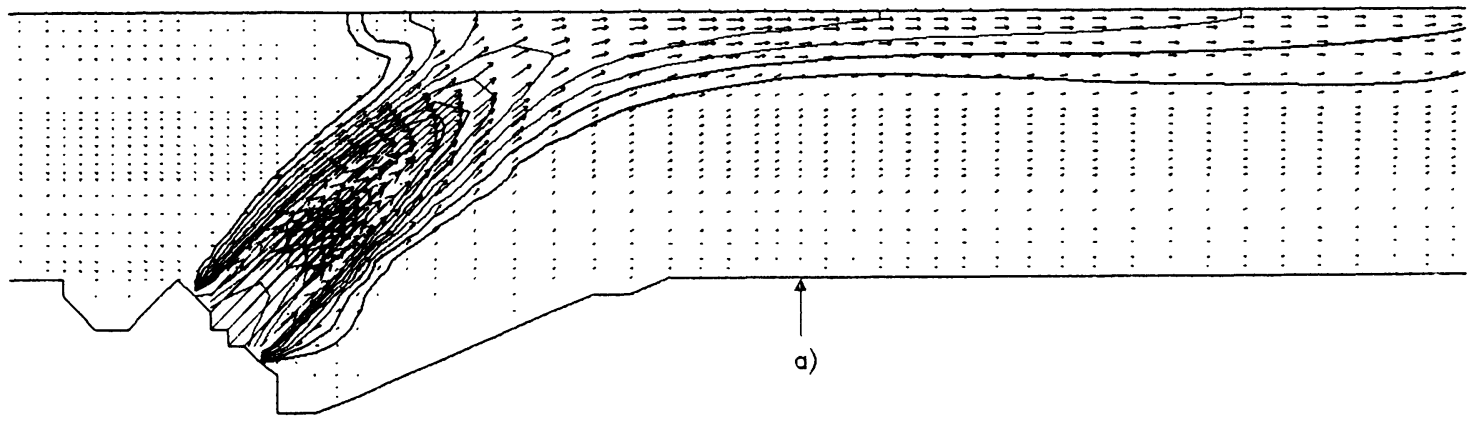

Fig. 9. - Expérimentation à l'échelle industrielle au laboratoire plasma des Renardières. Champs de vitesse et température calculés dans le plan de symétrie du calcul. Le maillage de calcul est porté figure 12. Le diamètre de conduit aval est de $0,28 \mathrm{~m}$.

[Experiment at industrial scale in the Renardieres laboratory. Computed velocity and temperature in the plane of symmetry. The computing grid is plotted in figure 12. The diameter of the downstream pipe is $0.28 \mathrm{~m}$.]
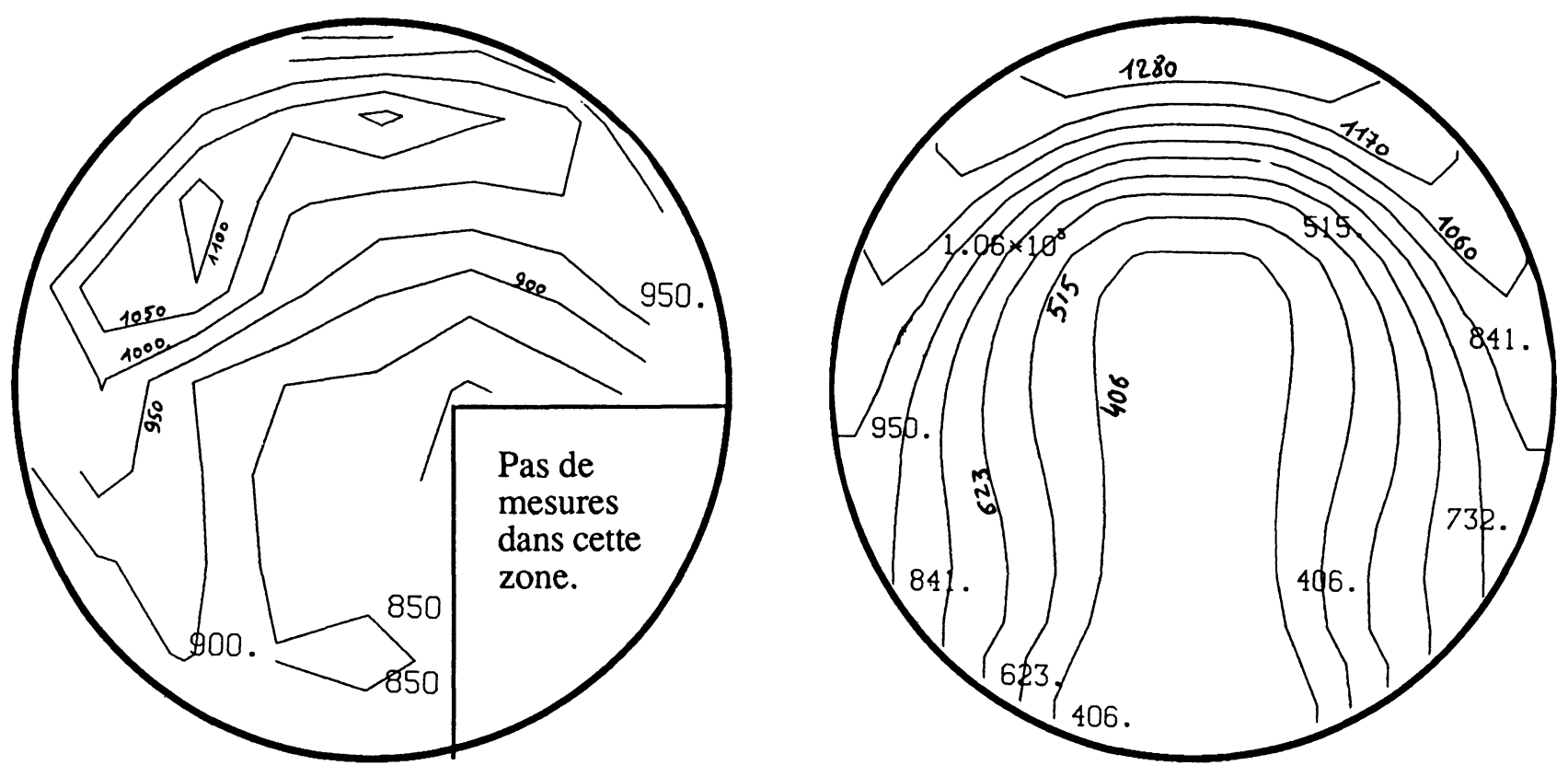

Fig. 10. - Cartes de température dans la section $a$ (située à $50 \mathrm{~cm}$ en aval torche) mesuree (à gauche) et calculee (à droite). [Plots of the temperature in section $a$ (situated $0.5 \mathrm{~m}$ downstream from the torch exit). Left : measured ; right : computed.] 

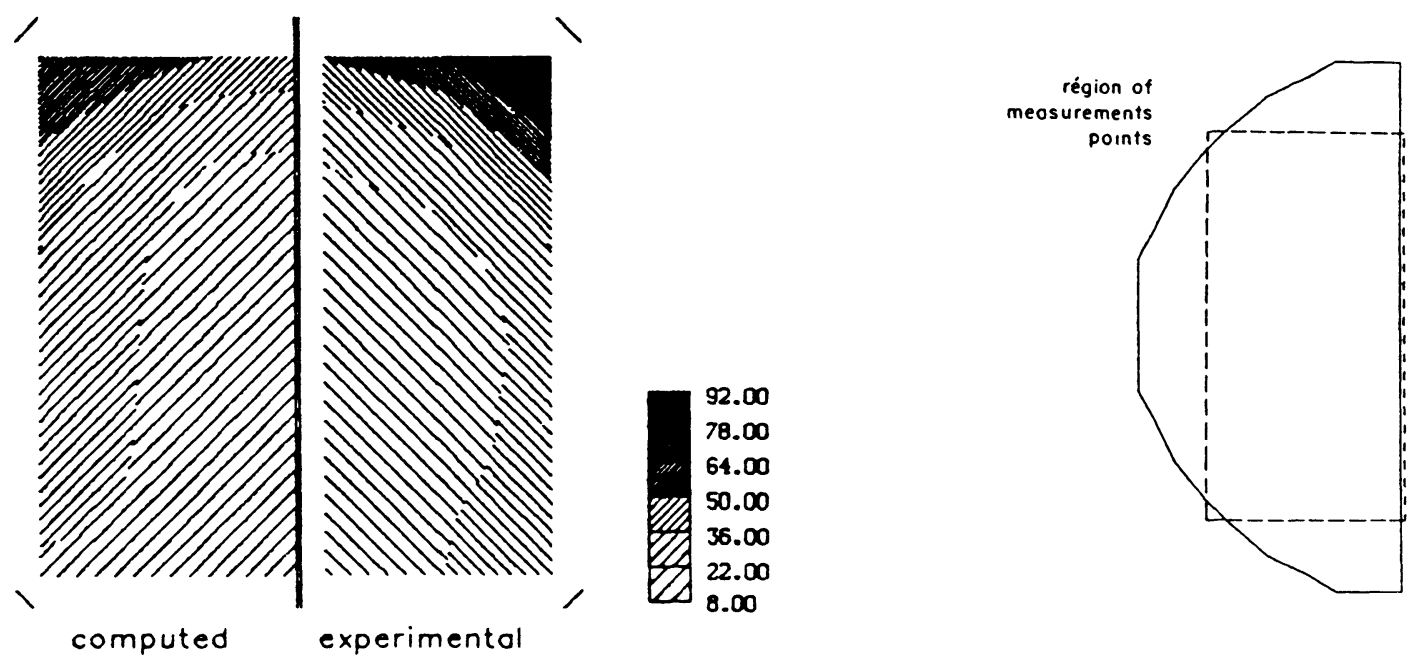

Fig. 11. - Cartes de vitesse longitudinale dans la section $a$ (située à $50 \mathrm{~cm}$ en aval de la sortie torche calculée (à gauche) et mesurée (à droite). Ces cartes de vitesse sont tracées ici dans le rectangle inscrit dans une demi-section de conduite.

[Plots of longitudinal velocity in section $a$ (situated $0.5 \mathrm{~m}$ downstream from the torch exit. Left : computed ; right : measured. These velocities are plotted in the rectangle inside the half pipe section (measuring area for velocity)].

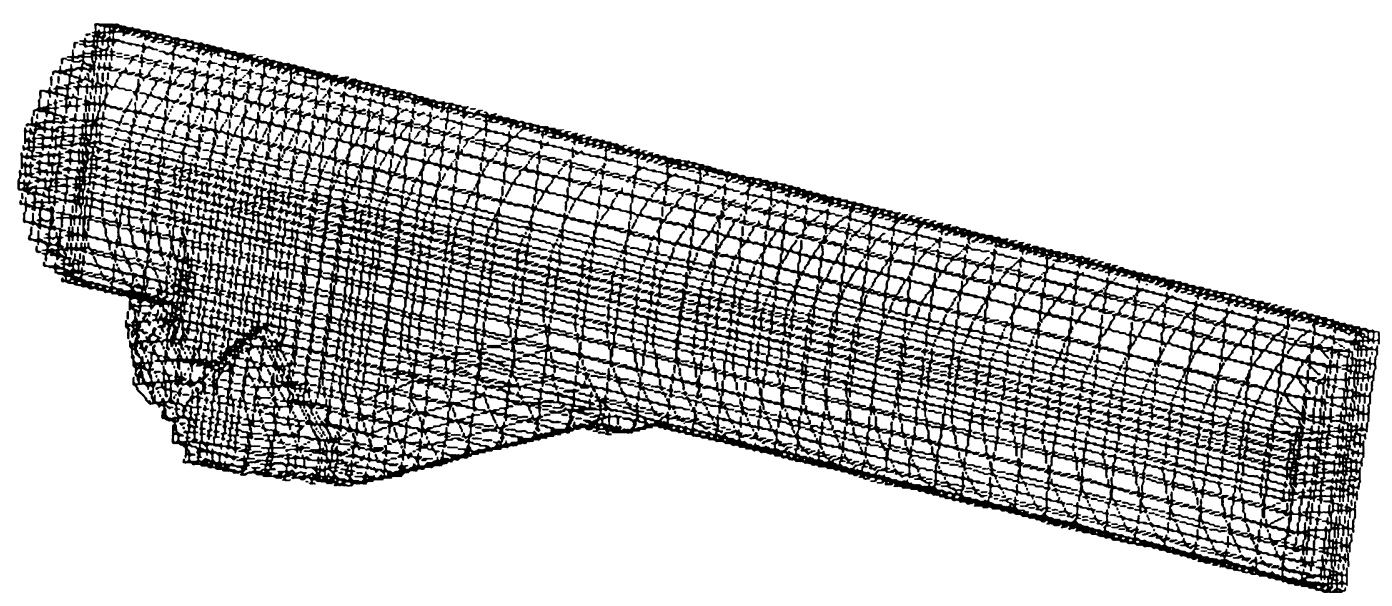

Fig. 12. - Vue tridimensionnelle du maillage de calcul de l'expérience à l'échelle industrielle du laboratoire plasma des Renardières.

[Three-dimensional view of the grid used to compute the Renardières industrial-scale experiment.]

3.3 EXEMPLE D'UN ÉCOULEMENT RÉACTIF DE COMPOSITION ATOMIQUE VARIABLE : CALCUL D'UNE FLAMME DE DIFFUSION TURBULENTE. - Le domaine des flammes turbulentes, dans lequel de nombreux résultats d'études expérimentales sont disponibles, se prète bien à une première évaluation de la capacité de l'hypothèse d'équilibre chimique à prédire la composition d'un mélange réactif complexe.

La figure 13 présente ainsi les fractions volumiques mesurées (Hassan, Lockwood, Moneib [24]) et calculées sur l'axe de symétrie d'une flamme de diffusion turbulente résultant de l'émission d'un jet de gaz naturel en air calme. Ce calcul fait suite à une serie antérieure de calculs sur la même flamme, calculs qui supposaient la chimie réduite à une réaction unique rapide (voir [3]). Dans le présent calcul, la composition complète du mélange est calculée à partir de données d'équilibre chimique (Prado [25]) et de la méthode exposée au paragraphe 2.3 (la composition du mélange est calculée en appliquant l'équation (20) à chaque espèce dominante). Les champs de température calculés sont proches de l'expérience et des résultats des calculs antérieurs [3]. Le pic de concentration volumique en $\mathrm{CO}$ est dans le calcul bien situé par rapport au pic de con- 


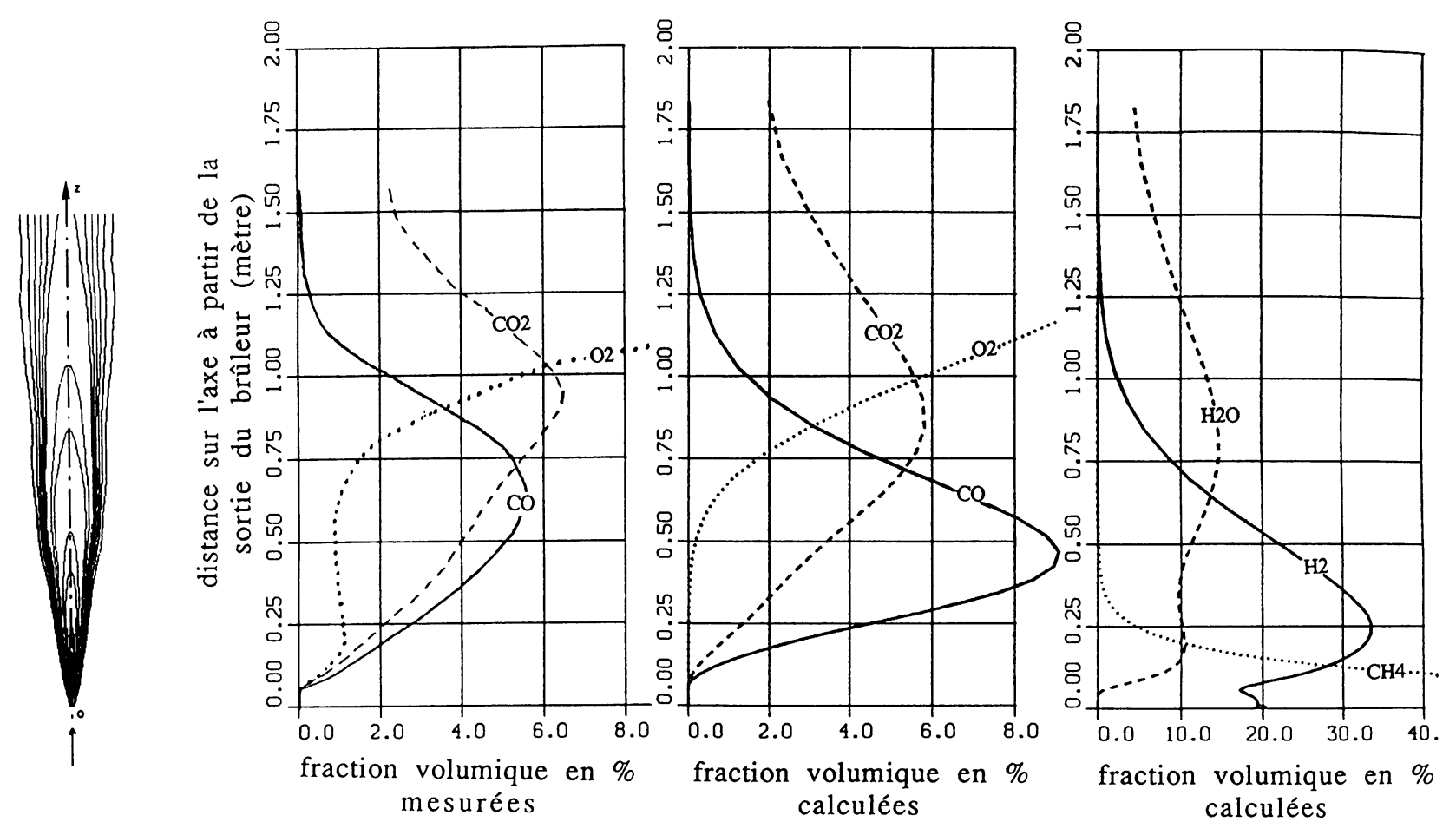

Fig. 13. - Calcul d'une flamme turbulente en utilisant l'hypothèse d'équilibre chimique instantané (Eq. (19, 20)). La flamme étudiée expérimentalement par Hassan, Lockwood et Moneib [24] résulte d'un jet de méthane en air calme, par une buse de $8 \mathrm{~mm}$ de diamètre, avec une petite flamme pilote d'hydrogène. Dans le présent calcul, la flamme pilote n'est pas représentée, et méthane et hydrogène sont supposés prémélangés $\left(20 \%\right.$ vol $\mathrm{H}_{2}+80 \%$ vol $\left.\mathrm{CH}_{4}\right)$. A gauche : isothermes calculées dans le domaine $0<z<1,5 \mathrm{~m}$. A droite : profils de concentration volumique mesurés [23] et calculés sur l'axe oz de la flamme.

[Computation of a turbulent flame using the instantaneous equilibrium assumption (Eq. $(19,20)$ ). The flame studied experimentally by Hassan, Lockwood and Moneib [24] results from a methane jet discharged in open air, with a small hydrogen pilot flame. In the present computation, the pilot flame is not represented (methane and hydrogen are assumed to be premixed with $20 \%$ vol $\mathrm{H}_{2}$ and $80 \%$ vol $\mathrm{CH}_{4}$ ). Left : computed isothermal lines in the area $0<z<1.5 \mathrm{~m}$. Right : computed (present study) and measured [24] mean volumic concentrations along the axis $0 z$ of the flame.]

centration en $\mathrm{CO}_{2}$, mais de trop grande amplitude par rapport à l'expérience. L'existence dans les mesures d'oxygène à ce niveau indique sans doute que l'hypothèse de cinétique rapide n'est pas très valide dans cette zone (la vitesse d'ecoulement est forte). Dans la partie supérieure de la flamme, les concentrations mesurées et calculées concordent bien. On observe dans les résultats de calcul une décomposition rapide du méthane liée à une importante production d'hydrogène.

\section{Conclusion.}

Parmi les difficultés de modélisation des écoulements de plasma thermiques en extinction, la turbulence et son intéraction avec les phénomènes réactifs est une des plus aigues. Dans le cas où plasma et courant de dilution sont produits à partir du même gaz, cas qui minimise cette difficulté, une démarche de validation de la modélisation numérique a été effectuée. Cette démarche comprend des cas de validation en écoulement incompressible, en écoulement non réactif à masse volumique variable, enfin des cas de mélange plasmacourant de dilution. Les résultats présentés permettent de juger de la concordance obtenue entre résultats expérimentaux et résultats de calcul, pour ce type d'écoulement qui présente des difficultés de modélisation en même temps que de métrologie.

Dans le cas des réacteurs chimiques à plasma, le problème de l'interaction entre la turbulence et les réactions chimiques se pose de façon plus aigue, et nous avons tenté ici de discuter de quelques aspects de ce problème. Les résultats présentés sur une flamme turbulente suggèrent que l'hypothèse d'équilibre chimique instantanné peut fournir des éléments intéressants sur la composition d'un mélange réactif, si l'on prend des précautions sur l'exploitation des résultats dans les zones où les temps caractéristiques de la chimie deviennent du même ordre que ceux de l'écoulement (par exemple, zones de trempe dans le cas d'un réacteur chimique à plasma).

L'étude de la colonne d'arc, des transferts radiatifs, des réacteurs chimiques ou des problèmes de combustion assistée par plasma fonctionnant avec une phase solide, enfin qui n'ont pas été évoqués dans le présent article, sont également des directions de recherches dans lesquelles un effort de modélisation se poursuit. 
Bibliographie

[1] Simonin O., Viollet P. L., Numerical study on phase dispersion mecanisms in turbulent bubbly flows, Int. Conf. on Mechanics of Two-Phase Flows, Taiwan (June 1989).

[2] BORGHI R., POURBAIX E., La Recherche Aérospatiale 4 (1983) 245-255.

[3] Viollet P. L., GaRReton D., Mechitoua N., Computation of turbulent diffusion flames with radiation, Proc. rencontre SFT 87, Ecole Centrale des Arts et Manufactures (Mai 1987).

[4] LAUNDER B. E., SPALDING B., The numerical computation of turbulent flows, Comp. Meth. Appl. Mech. Eng. vol. 3 (1974).

[5] HUANg P. G., LeChZINER M., Stabilization of recirculating flow computations performed with secondmoment closure and third-order discretization, 5th Symp. on Turbulent Shear Flow, Ithaca N.Y. (Aout 1985).

[6] VANDROMME D., HA MINH H., About the coupling of turbulence closure models with averaged NavierStokes equations, J. Computational Phys. (Aout 1986).

[7] KANNiChE M., BARON F., Viollet P. L., Second moment closure prediction of recirculating flow and of countergradient heat flux in stratified flow, 7th Int. Symp. on Turbulent Shear Flows, Stanford (Aug. 21-23, 1989).

[8] Dewagenaere Ph., Esposito P., Lana F., Viollet P. L., Three dimensional computations of nonisothermal wall-bounded complex flows, 9 th Int. Conf. on Numerical Methods in Fluid Dynamics, Saclay (Juin 1984).

[9] EsPosiro P., Résolution des équations de transport par la méthode des caractéristiques, rapport EDFLNH HE 41/81.16.

[10] GabillaRd M., Viollet P. L., The numerical computation of secondary flow and density currents in a curved pipe, proc. 3rd Int. Symp. on Refined Flow Modelling and Turbulence Measurements, Tokyo (July, 1988).

[11] Viollet P. L., Modélisation numérique du mélange d'un jet de plasma en extinction dans un écoulement froid en conduite, EDF. Bulletin de la Direction des Etudes et Recherches, Série A, $n^{\circ} 2$, (1985) pp. 7-32.

[12] HERARD J. M., Modélisation et calcul d'écoulements turbulents isothermes de gaz à densité variable,
Thèse Institut National Polytechnique de Toulouse, Mars 1986.

[13] Chassaing P., Herard J. M., Second-order modelling of variable density turbulent mixing, Proc. 6th Symp. on Turbulent Shear Flow, Toulouse (Sept 1987).

[14] JONES W. P., PASCAU A., Calculation of variable density flow with a second moment closure, Euromech 237, Marseille (Juillet 1988) pp. 49-53.

[15] ANSELMET F., SCHIESTEL R., BAHAROUI E. M., ZAHIBO I., FULACHIER L., Experimental investigation and turbulent flux modelling of a strongly heated turbulent jet, Euromech 237, Marseille (Juillet 1988) pp. 33-37.

[16] MAGRe P., MOREAU P., COllin G., BORGHI R., PÉAlat M., Further studies by CARS of premixed turbulent combustion in a high velocity flow, Combustion and Flame 71 : 147-168 (1988).

[17] GABILlARD M., Flamain D., résultats non publiés (1989).

[18] GabillaRD M., DURANTE N., résultats non publiés (1989).

[19] Viollet P. L., Flamain D., Lana F., Olivier J. C., Etude du mélange d'un jet émis à $45^{\circ}$ dans un écoulement gazeux plus dense, Rapport EDF HE44/87.22 (Oct 1988).

[20] SIMONIN O., BÉCHART J., résultats non publiés (1987).

[21] DE IZARRA C., Contribution à l'étude de colonnes de plasma d'argon dans un domaine étendu de temperature, Thèse, université d'Orléans, Novembre 1988 .

[22] LANA F., Viollet P. L., KasabJi F., Mélange d'un jet de plasma dans un écoulement froid : validation des calculs tridimensionnels et applications industrielles, Proc. 8th Int. Symp. on Plasma Chemistry, Tokyo (Juillet 1987, 31-36) (rapport EDF HE44/87.35).

[23] CAHEN F., SPiBerg P., Deschamps P., Kassabji F., Metrologie optique de la torche à plasma des Renardières ; mesures de température par DRASC, Rapport EDF HP/119/88/008.

[24] PRADO G., communication personnelle.

[25] HASSAN M., LOCKWOOD F., MONEIB H., Fluctuating temperature and mean concentration measurements in a vertical turbulent free jet diffusion flame, La Rivista dei Combustibli 34 fasc. 9-12 (Sept. 1980) 357-372. 